\title{
La mise en œuvre d'un transfert organisationnel : dynamique des approches collaboratives
}

\author{
Annick Trépanier, M. Sc., chef de service \\ Centre de santé et de services sociaux de Chicoutimi \\ Brahim Meddeb, Ph. D., professeur \\ Université du Québec à Chicoutimi
}

\section{INTRODUCTION}

\section{L'engagement, l'utilisation du personnel en place et la valorisation de leur expertise se sont avérés d'une importance capitale dans la conception et la mise en ouvre de modèles d'organisation et dans \\ l'opérationnalisation de ce nouveau secteur d'activité, soit les services jeunesse.}

Au cours des années 2008-2010, suite à de nombreuses discussions et réflexions, animées par les directions de l'Agence de la santé et des services sociaux, du centre de réadaptation en dépendances, des Centres Jeunesse et des directeurs des centres de santé et services sociaux (CSSS) de la région du Saguenay-Lac-SaintJean, il fut décidé d'intégrer les services de la clientèle jeunesse au Centre de Réadaptation en Dépendance (CRD) du CSSS de Jonquière. Le but de cette intégration est d'assurer l'accessibilité et la qualité des services en dépendance jeunesse.

La logique de la dispensation de services aux clientèles adulte et jeune regroupées dans un même centre nécessitait alors un travail préparatoire colossal. En effet, le contexte de changement dans lequel s'est effectuée la réorganisation touchait à la fois la clientèle, les intervenants et les organisations impliquées dans le transfert. Au cœur de cette intervention, l'engagement, l'utilisation du personnel en place et la valorisation de leur expertise se sont avérés d'une importance capitale dans la conception et la mise en œuvre de modèles d'organisation et dans l'opérationnalisation de ce nouveau secteur d'activité, soit les services jeunesse.
Puisque le service en dépendance aux jeunes de la région du Saguenay-Lac-Saint-Jean était antérieurement desservi par les Centres Jeunesse (CJ), le CRD était le seul centre de l'association des centres de réadaptation en dépendance du Québec à se démarquer par l'absence de service pour la clientèle jeunesse (12-18 ans). L'arrivée de cette nouvelle clientèle oblige donc l'établissement du CSSS de Jonquière à réorganiser ses services. Un transfert harmonieux et efficient s'impose en déployant les ressources humaines, physiques, matérielles et informationnelles nécessaires, tout en assurant l'accessibilité et la qualité des services pour la clientèle et les partenaires. Pour mener à bien ce changement, il a été approprié d'accompagner le CRD dans la conception d'un modèle d'organisation du service à la clientèle jeunesse. Sur ce point, l'effort consistait alors à mettre en place un processus d'accueil et d'intégration des nouveaux intervenants et de ceux des équipes territoriales desservant les clientèles jeunes et adultes. C'est dans cette perspective que ce texte présente les principales activités ayant permis la réorganisation des services suivant des approches collaboratives.

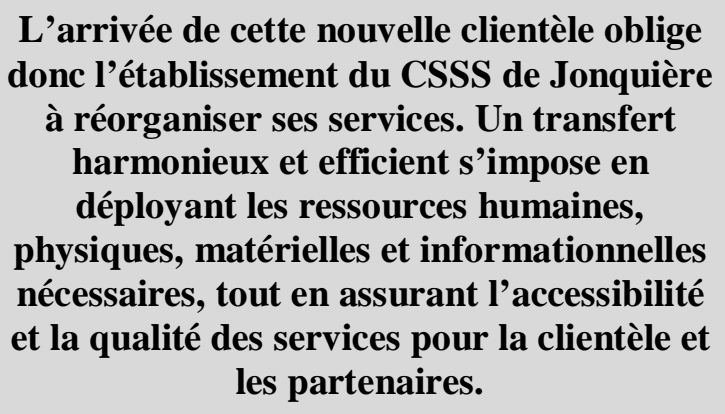

L'arrivée de cette nouvelle clientèle oblige donc l'établissement du CSSS de Jonquière à réorganiser ses services. Un transfert harmonieux et efficient s'impose en déployant les ressources humaines, physiques, matérielles et informationnelles nécessaires, tout en assurant l'accessibilité et la qualité des services pour la clientèle et les partenaires. 


\section{CADRE CONCEPTUEL DE L'INTERVENTION}

\section{La communication efficace serait propice à l'implication des acteurs concernés, une implication qui accroît les probabilités de succès d'une transformation organisationnelle.}

Avant d'examiner le contenu détaillé de l'intervention, il convient de présenter le cadre conceptuel élaboré et mis en œuvre dans le cadre de cette réorganisation. La restructuration des services s'est basée sur l'approche systémique en gestion des ressources humaines, telle qu'illustrée à la figure 1 . Ce cadre conceptuel met en relief l'importance de plusieurs acti- vités dont l'accueil et le développement des compétences. Tout au long du processus visant la mise en place de ces activités en vue d'atteindre les résultats, il est essentiel de miser sur la communication à chacune des actions. C'est pourquoi il est jugé pertinent d'intégrer la communication en tant que dimension pouvant influencer les activités productives, mais aussi l'évaluation de l'atteinte des résultats. En effet, la communication efficace serait propice à l'implication des acteurs concernés, une implication qui accroît les probabilités de succès d'une transformation organisationnelle.

Figure 1 - Approche systémique en gestion des ressources humaines

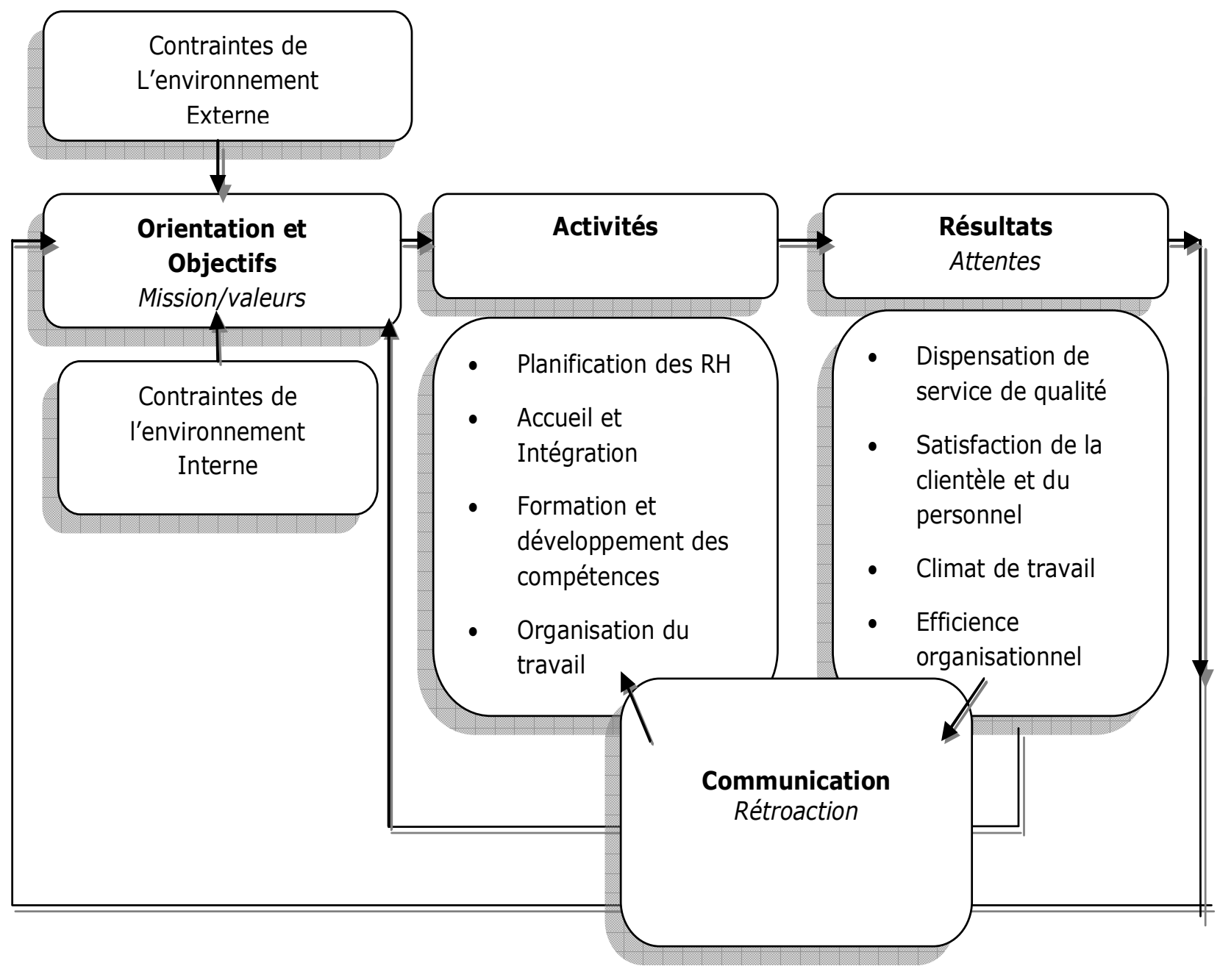


L'approche systémique accorde une attention particulière à la capacité organisationnelle suivant laquelle l'organisation combine ses ressources productives et met en œuvre des activités de gestion ressources humaines de façon alignée à la mission et aux objectifs stratégiques.

L'approche systémique accorde une attention particulière à la capacité organisationnelle suivant laquelle l'organisation combine ses ressources productives et met en œuvre des activités de gestion des ressources humaines de façon alignée à la mission et aux objectifs stratégiques. Plus spécifiquement, elle met en relief l'importance de la formation et le développement des compétences, mais aussi des méthodes d'apprentissage. En s'appuyant sur les fondements méthodologiques de l'apprentissage, la formation par les pairs peut être extrêmement efficace sans pour autant entraîner des coûts trop élevés. À court terme, elle permet à l'organisation de développer sa capacité à répondre aux besoins de connaissances de base des deux clientèles et de permettre la polyvalence des ressources humaines. La formation par les pairs a été conçue sur la base de quelques approches théoriques ${ }^{1}$. D'abord, la théorie de l'apprentissage social stipule que les gens sont des modèles de comportements humains et que certaines personnes ont la capacité de provoquer un changement de comportements chez certains individus. Ensuite, l'approche théorique de l'action raisonnée indique que la perception qu'a une personne influe sur ses actions. Également, la théorie de diffusion de l'innovation postule que les leaders d'opinion peuvent en diffusant de l'information changer des comportements et influencer les normes du groupe. La facilitation des apprentissages significatifs repose sur la relation entre l'animateur et l'apprenant, mais aussi sur la participation active de l'apprenant. Enfin, la théorie de l'éducation participative a joué un rôle majeur dans le développement de l'éducation par les pairs.

Grâce à ce cadre conceptuel, il a été possible de réaliser l'intégration organisationnelle suivant une méthodologie spécifique et un plan d'activité précis. En effet, afin de concrétiser les activités à réaliser et de planifier le bon déroulement, un plan d'action répondant de façon détaillée et concrète à chacun des objectifs du mandat a été élaboré et mis en œuvre. Pour ce faire, ce plan a servi de balise par la définition de tâches ordonnancées de façon logique et encadrées d'un délai prévu de réalisation, tel que présenté au tableau 1.

La formation par les pairs peut être extrêmement efficace sans pour autant entraîner des coûts trop élevés. À court terme, elle permet à l'organisation de développer sa capacité à répondre aux besoins de connaissances de base des deux clientèles et de permettre la polyvalence des ressources humaines.

Tableau 1 - Plan d'action « Arrimage de la clientèle Jeunesse CRD »

\begin{tabular}{|c|l|l|l|l|l|l|}
\hline \multicolumn{7}{|c|}{ ANALYSE DE LA PROBLÉMATIQUE } \\
\hline $\begin{array}{c}\text { OBJECTIFS } \\
\begin{array}{c}\text { Résultats } \\
\text { attendus }\end{array}\end{array}$ & $\begin{array}{c}\text { ACTIONS/MOYENS } \\
\text { Quoi? }\end{array}$ & INDICATEURS & $\begin{array}{c}\text { RESPONSIBLE } \\
\text { Qui? }\end{array}$ & $\begin{array}{c}\text { ÉTAPES DE } \\
\text { RÉALISATION } \\
\text { Comment? }\end{array}$ & $\begin{array}{c}\text { ÉCHÉANCES } \\
\text { D'ÉTAPE } \\
\text { Date }\end{array}$ & ÉVALUATION \\
\hline & 1. & & & & Atteint ou \\
\hline & & 2. & & & Non atteint \\
\hline
\end{tabular}




\section{MISE EN GEUVRE DU TRANSFERT ORGANISATIONNEL}

\author{
La mission du CRD consiste à offrir des \\ services d'adaptation ou de réadaptation et \\ d'intégration sociale à des personnes ayant \\ des problèmes d'alcoolisme, de toxicomanie \\ ou autres dépendances.
}

La mission du CRD consiste à offrir des services d'adaptation ou de réadaptation et d'intégration sociale à des personnes ayant des problèmes d'alcoolisme, de toxicomanie ou autres dépendances. La réalisation de cette mission se déroule dans un environnement dont les composantes sont variées: les règles gouvernementales, les lois, l'offre de service de base en toxicomanie et la gestion axée sur les résultats. Ces paramètres poussent à uniformiser et à appliquer des pratiques gagnantes. De plus, elles facilitent la planification de la gestion des ressources humaines et toutes les activités qui en découlent. Les besoins spécifiques et complexes de la clientèle en dépendance déterminent les exigences à respecter sur le plan de l'environnement interne dont les principaux actifs sont les connaissances et les habiletés des employés. Compte tenu de la nature des services offerts, les compétences humaines sont primordiales et constituent une ressource vitale.

Pour préserver ces compétences, l'intégration à l'équipe existante des intervenants ayant de l'expertise auprès de la clientèle jeunesse a été prévue dans cette expérience de transfert organisationnel. Cette intégration repose sur les prémisses et finalités de la gestion des ressources humaines : "Disposer à temps, en effectifs suffisants, et en permanence, des personnes compétentes et motivées, pour effectuer le travail nécessaire en les mettant en situation de valoriser leurs talents avec un niveau élevé de performance et de qualité, à un coût salarial compatible avec ses objectifs économiques, et dans le climat social le plus favorable possible. ${ }^{2}$

\subsection{Accueil et intégration des nouveaux intervenants : une responsabilité partagée}

En misant sur la collaboration comme philosophie d'intervention, l'implication de tous les employés a contribué à la façon d'atteindre les objectifs fixés. L'implantation des services jeunesse en dépendance au CRD du CSSS de Jonquière s'est faite selon le cadre conceptuel à partir des objectifs à atteindre, de la définition des besoins et de l'analyse des ressources nécessaires à la réussite de cette intégration aux services actuels.

L'élaboration de la procédure d'accueil se veut un moyen de favoriser l'intégration, l'engagement, le confort à exercer ses tâches, mais aussi d'encourager la rétention des nouveaux employés dans l'organisation. Dans le cadre de l'intégration jeunesse, des intervenants du CJ et plusieurs autres intervenants ont joint l'équipe du CRD. Suite à ce transfert, l'accueil et l'intégration se voulait un moment privilégié de contact avec leur nouvelle organisation. Les nombreux outils développés et mis en place lors de ce mandat démontrent l'importance de l'uniformité de l'information véhiculée lors de ce processus d'accueil-intégration, mais aussi de la reconnaissance du CRD envers ses ressources humaines. Dès l'accueil du nouvel employé au $\mathrm{CRD}$, un programme de parrainage a aussi été instauré afin de favoriser l'intégration et l'acquisition de nouvelles connaissances en lien avec la fonction et l'organisation. Le parrainage répond à un besoin momentané d'enrichissement du savoir-faire des intervenants. L'accueil, l'intégration et le parrainage sont des activités différentes, mais interdépendantes. Elles assurent une fusion complète et réussie de l'employé dans l'organisation. Chacune de ses activités poursuit des objectifs spécifiques, interdépendants et complémentaires.

\section{L'accueil, l'intégration et le parrainage sont des activités différentes, mais \\ interdépendantes. Elles assurent une fusion complète et réussie de l'employé dans \\ l'organisation. Chacune de ses activités poursuit des objectifs spécifiques, interdépendants et complémentaires.}

En outre, pour uniformiser la compréhension du processus, une grille de répartition des responsabilités a été aussi développée. Cet outil de 
gestion, présenté au tableau 2, définit qui doit agir à titre de responsable $(\mathrm{R})$ ou de support $(\mathrm{S})$ lors de l'accueil $^{3}$. Enfin, certaines cellules fusionnées démontrent le partage des responsabilités. L'application de cette grille intégrée dans le processus d'accueil du CRD est d'une grande efficacité, car elle a permis l'atteinte des objectifs des premières journées : accueillir, informer et organiser tout en finalisant la partie administrative de l'embauche.

Tableau 2 - La répartition des responsabilités en matière d'accueil au CRD

\begin{tabular}{|c|c|c|c|}
\hline Responsabilités & $\begin{array}{c}\text { Direction } \\
\text { Chef de } \\
\text { programme }\end{array}$ & $\begin{array}{l}\text { Coordonnateur } \\
\text { clinique }\end{array}$ & Collègues \\
\hline 1. Préparation de l'arrivée & & & \\
\hline Confirmation de date d'arrivée & $\mathrm{R}$ & S & \\
\hline Préparation de la pochette d'accueil & & $\mathrm{R}$ & \\
\hline Préparation de l'espace de travail & $\mathrm{R}$ & S & \\
\hline Préparation des outils de travail & & S & \\
\hline Annonce de l'arrivée aux collègues & \multicolumn{2}{|c|}{ Rou S } & \\
\hline \multicolumn{4}{|l|}{ 2. Première journée } \\
\hline Accueil de l'employé & S & $\mathrm{R}$ & \\
\hline $\begin{array}{l}\text { Remise de la pochette des documents } \\
\text { d'accueil }\end{array}$ & & $\mathrm{R}$ & \\
\hline Présentation de l'organisation & $\mathrm{R}$ & S & \\
\hline Visite du milieu de travail & \multicolumn{2}{|c|}{ R ou S } & S \\
\hline Présentation aux collègues & & $\mathrm{R}$ & $S$ \\
\hline Désignation de l'espace de travail & & $\mathrm{R}$ & \\
\hline $\begin{array}{l}\text { Explication sur le contenu du poste, } \\
\text { attentes }\end{array}$ & S & $\mathrm{R}$ & \\
\hline \multicolumn{4}{|l|}{ 3. Suivi de l'Accueil } \\
\hline Évaluation du programme d'Accueil & $\mathrm{R}$ & & \\
\hline Évaluation du besoin de formation & S & $\mathrm{R}$ & \\
\hline \multicolumn{4}{|l|}{ 4. Au cours des deux premières semaines } \\
\hline $\begin{array}{l}\text { Expliquer les communications internes } \\
\text { (réunion) }\end{array}$ & & $\mathrm{R}$ & $S$ \\
\hline Confirmer la compréhension des attentes & S & $\mathrm{R}$ & \\
\hline R: Responsable & & & \\
\hline
\end{tabular}


Cette réflexion de répartition entre les acteurs impliqués dans l'accueil a permis d'établir une liste d'informations représentée au tableau 3. Cet outil a pour objectif d'éviter de surinformer le nouvel employé à son arrivée. Ainsi, toutes les informations pertinentes à recueillir étant déterminées, l'employé pourra lui-même en prendre connaissance lors de la période d'intégration. L'employé est aussi responsable de son apprentissage par la voie de sa propre participation aux différentes activités du processus d'accueil. Il partage donc la responsabilité de collecte d'informations par le biais de cette liste. Cet outil a été mis en œuvre et évalué positivement par les responsables de l'accueil-intégration et par les employés intégrés. À partir des éléments de cette liste, un cartable d'accueil contenant tous les documents et outils nécessaires a été élaboré. $\mathrm{Sa}$ mise en œuvre permet aussi l'uniformité du processus d'accueil de la personne et la transmission intégrale des informations pour tous les employés embauchés.

\section{Tableau 3 - Accueil-intégration une responsabilité partagée «Une responsabilité partagée! Ais-je entendu parler de ces éléments? » Intervenant en toxicomanie}

\begin{tabular}{|c|c|c|}
\hline Nom & Entrée en $\mathrm{F}$ & ste : \\
\hline \multicolumn{3}{|c|}{ Point de services : } \\
\hline FAIT & ÉLÉMENT DE L'ACCUEIL ET INTÉGRATION & À COMPLÉTER/COMMENTAIRE \\
\hline$\square$ & Rencontre avec les Ressources Humaines CSSSJ & $\square$ \\
\hline \multicolumn{3}{|c|}{ Environnement } \\
\hline$\square$ & Présentation de l'équipe & $\square$ \\
\hline$\square$ & Visite du milieu de travail & $\square$ \\
\hline \multicolumn{3}{|c|}{ Rencontre avec le coordonnateur/chef de programme (éléments à aborder) } \\
\hline$\square$ & Compréhension de la mission de l'organisation & $\square$ \\
\hline$\square$ & Organigramme de l'organisation & $\square$ \\
\hline$\square$ & Attente du nouvel employé face à l'organisation & $\square$ \\
\hline$\square$ & Attitrer un bureau et donner clef & $\square$ \\
\hline $\bar{\square}$ & Carte d'employé et de stationnement & $\bar{\square}$ \\
\hline$\square$ & Attentes de l'organisation face à la personne & $\square$ \\
\hline$\square$ & Processus d'évaluation pour l'obtention de la probation (déterminer une date) & $\square$ \\
\hline$\square$ & Horaires de travail & $\square$ \\
\hline$\square$ & Processus d'accueil de la clientèle & $\square$ \\
\hline $\bar{\square}$ & Planifier date de formation du serveur & $\square$ \\
\hline$\square$ & Procédure d'utilisation de la boîte vocale & $\square$ \\
\hline$\square$ & Feuilles de temps et comptes de dépenses & $\square$ \\
\hline$\square$ & Remettre liste téléphonique CRD/ CSSSJ / Partenaires & $\square$ \\
\hline \multicolumn{3}{|c|}{ Lectures à faire } \\
\hline$\square$ & Guide d'accueil & $\square$ \\
\hline$\square$ & Offre de Service CRD & $\square$ \\
\hline$\square$ & Politique de confidentialité & $\square$ \\
\hline \multicolumn{3}{|c|}{ Codes nécessaires à l'exercice de ses fonctions : } \\
\hline$\square$ & Serveur et adresse courriel & $\square$ \\
\hline$\square$ & Code téléphonique/télécopieur (interurbain)/photocopieur & $\square$ \\
\hline \multicolumn{3}{|c|}{ Projets spéciaux } \\
\hline$\square$ & Processus d'amélioration continue de qualité/sécurité d'Agrément Canada & $\square$ \\
\hline$\square$ & Informations sur les programmes des partenaires & $\square$ \\
\hline \multicolumn{3}{|c|}{ Parrainage } \\
\hline$\square$ & Orientation & $\square$ \\
\hline$\square$ & Programme de Parrainage du CRD & $\square$ \\
\hline \multicolumn{3}{|c|}{ Suivi de l'intégration avec le coordonnateur } \\
\hline$\square$ & S'assurer de l'appropriation de chaque élément mentionné ci haut & $\square$ \\
\hline$\square$ & Rencontre de suivi (date déterminée) & $\square$ \\
\hline
\end{tabular}


2.2 Développement des compétences et stratégie d'apprentissage

Outre la définition et la création d'outils, une des activités se situe dans l'élaboration d'un plan de formation. L'analyse de la littérature et l'identification des besoins du CRD ont permis de préciser les besoins de développement des compétences, de concevoir et de déployer les formations nécessaires. Les besoins de l'organisation de polyvalence et d'expertise confirment la nécessité du savoir spécialisé exigé pour les intervenants en réadaptation sur tout le territoire desservi. La formation vise donc à répondre aux besoins spécifiques. Elle cherche à outiller les intervenants et à leur offrir le support nécessaire afin de dispenser un service de qualité. Les compétences à développer prennent trois formes : transversale, fondamentale et spécialisée. C'est sur cette dernière que le modèle de formation par les pairs fut élaboré tout en s'assurant que les autres formes (compétences transversale et fondamentale) sont présentes et maîtrisées par les intervenants. La matrice, illustrée à titre d'exemple au tableau 4, représente le profil attendu des intervenants en toxicomanie en matière de compétences.

Tableau 4 - Matrice des compétences de l'intervenant en toxicomanie du CRD

\begin{tabular}{|c|c|c|c|}
\hline & \multicolumn{3}{|c|}{ FORMES } \\
\hline DIMENSION & TRANSVERSALE & FONDAMENTALE & SPÉCIALISÉE \\
\hline \multicolumn{4}{|c|}{ Connaître, comprendre ou maîtriser } \\
\hline Savoir & $\begin{array}{l}\text { - Informatique de base (Word, } \\
\text { courriel, etc.) } \\
\text { - Connaissance de la théorie en } \\
\text { lien avec les études en travail } \\
\text { social }\end{array}$ & $\begin{array}{l}\text { Organisation (mission, vision, } \\
\text { ressources, programmes) } \\
\text { - Processus inhérents au } \\
\text { développement du PSI, PI }\end{array}$ & $\begin{array}{ll}\text { - } & \text { Lois : } \\
\text { - } & \text { Outils : IGT adulte/ados, DEP-Ado } \\
\text { - } & \text { Pratiques gagnantes selon offre } \\
& \text { de service de base }\end{array}$ \\
\hline \multicolumn{4}{|c|}{ Mettre en œuvre ou savoir appliquer } \\
\hline Savoir-faire & $\begin{array}{l}\text { - Habiletés de compétence de } \\
\text { base } \\
\text { - Habiletés de communication } \\
\text { - Habiletés d'intervention }\end{array}$ & $\begin{array}{l}\text { - Évaluation psychosociale } \\
\text { - Planifier une intervention sociale } \\
\text { (selon clientèle) } \\
\text { - Réaliser une intervention sociale } \\
\text { - Etc. }\end{array}$ & $\begin{array}{l}\text { Évaluer en fonction de la } \\
\text { description des tâches } \\
\text { respectives (TS, TES, PSV) }\end{array}$ \\
\hline \multicolumn{4}{|c|}{ Savoir-vivre avec les autres } \\
\hline $\begin{array}{c}\text { Savoir-être } \\
\text { Et } \\
\text { Agir }\end{array}$ & - Compétences personnelles & \multicolumn{2}{|c|}{$\begin{array}{l}\text { Le savoir-être étant une compétence transversale, ne peut avoir une forme } \\
\text { de base ou spécialisée. }\end{array}$} \\
\hline
\end{tabular}

L'opérationnalisation efficace des compétences exige un niveau de motivation et une culture organisationnelle qui lui sont propices. L'efficacité est donc le résultat d'une triade $\mathrm{E}=\mathrm{mc}^{2}$ (Motivation X Compétences X Culture organisationnelle). Cette association est plus que jamais indispensable au sein d'une organisation apprenante ${ }^{4}$. Le personnel détenteur de savoirs doit être disposé et encouragé à le transmettre. La culture organisationnelle doit être axée sur la transparence, sur la communication et sur le partage des informations et des connaissances. Puisque la formation par les pairs motive les participants tout en développant la culture de transmission des compétences, elle a donc été privilégiée.

Le personnel détenteur de savoirs doit être disposé et encouragé à le transmettre. La culture organisationnelle doit être axée sur la transparence, sur la communication et sur le partage des informations et des connaissances. 
La stratégie d'apprentissage repose sur la formation «par les pairs » et le parrainage. Ces deux méthodes sont retenues, car elles permettent d'optimiser l'apprentissage et la rétention de l'information transmise. La formation par les pairs, suppose donc une approche, un canal de communication, une méthodologie, une philosophie et une stratégie. C'est dans l'esprit de collaboration et d'implication qu'a émergé l'idée de la formation par les pairs, d'autant plus que des intervenants du CJ possédant une expertise auprès de la clientèle jeunesse se greffaient à l'équipe.
L'utilisation de ces experts, nouvellement accueillis comme pairs formateurs, bonifiait du même coup leur intégration au CRD. Élaborée aux fins de ce projet, la dynamique d'apprentissage par les pairs se veut une formation qui propose l'enseignement et le partage d'expertise d'un (des) collègue (s) à un autre. Ce modèle fut donc le système d'apprentissage approprié à la situation afin de répondre aux besoins de l'organisation en fonction des objectifs à atteindre, tel que schématisé à la figure 2.

\section{Figure 2 - Modèle d'apprentissage par les pairs}

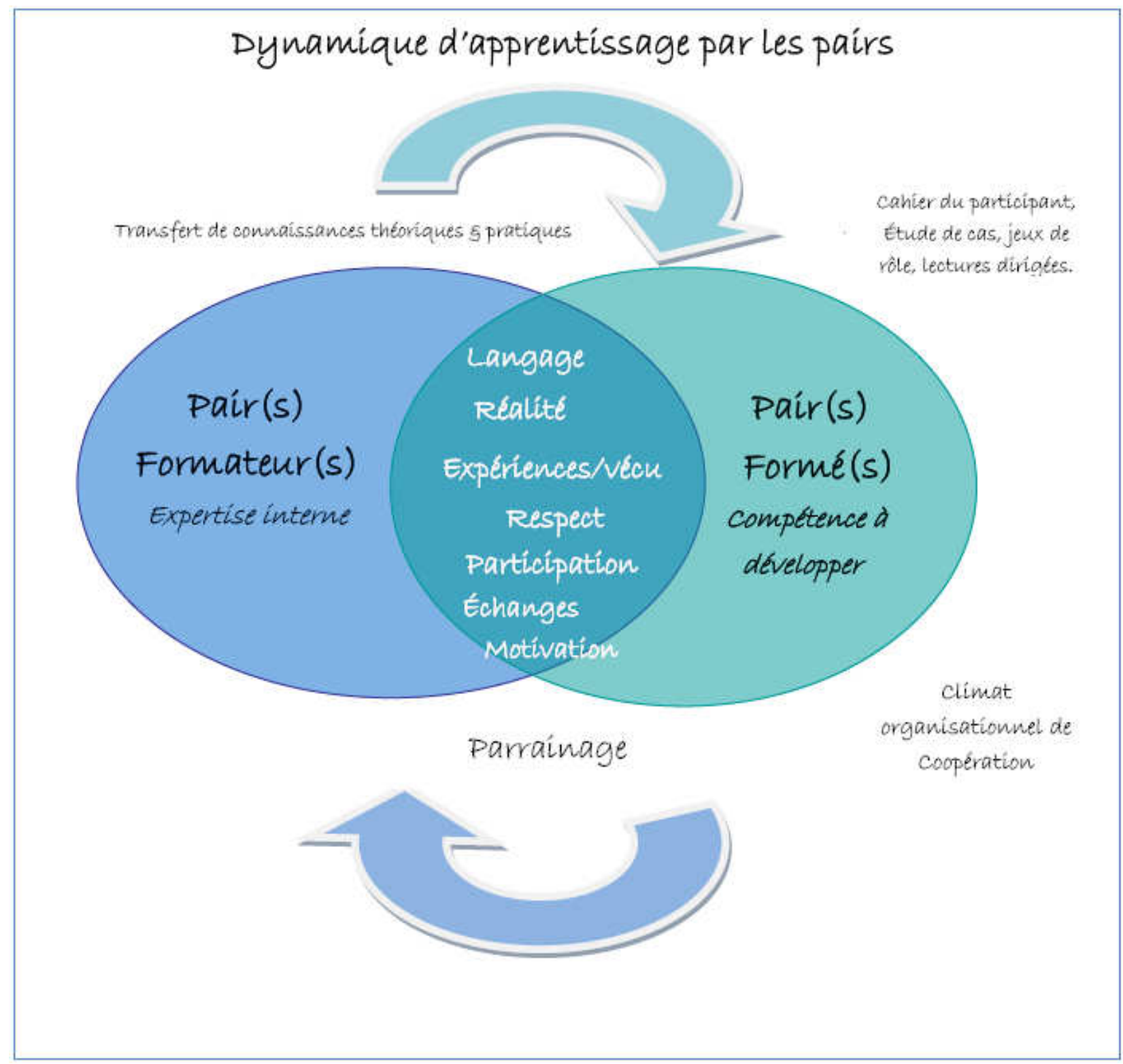


L'impact positif de cette stratégie d'apprentissage est donc ce processus horizontal de pairs égaux formateurs à l'origine de partage d'expériences pratiques, d'expertise et de contenus théoriques permettant rapidement aux intervenants d'appliquer les connaissances. La formation « par les pairs » permet de mettre en place un cadre favorisant le développement d'un esprit d'équipe et d'une fraternité créatrice.

Les «pairs formateurs» sont une force collective de l'organisation. Il faut donc capitaliser et tirer profit de leur connaissance, leur créativité et leur énergie afin de stimuler l'action collective. Ce dialogue entre intervenants permet d'établir un langage commun, d'intégrer le contenu et de développer le savoir-faire, le savoir-être et le savoir-agir. L'impact positif de cette stratégie d'apprentissage est donc ce processus horizontal de pairs égaux formateurs à l'origine de partage d'expériences pratiques, d'expertise et de contenus théoriques permettant rapidement aux intervenants d'appliquer les connaissances. La formation «par les pairs » permet de mettre en place un cadre favorisant le développement d'un esprit d'équipe et d'une fraternité créatrice.

\subsection{Parrainage « savoir-faire et faire-savoir »}

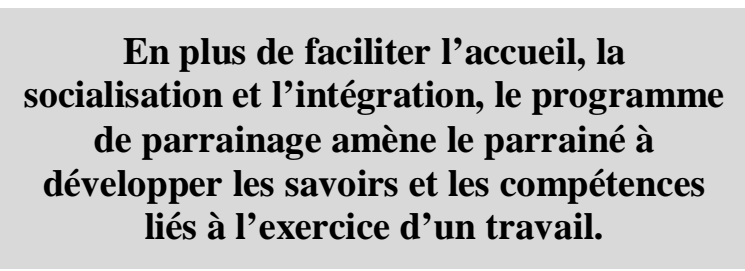

En plus de faciliter l'accueil, la socialisation et l'intégration, le programme de parrainage amène le parrainé à développer les savoirs et les compétences liés à l'exercice d'un travail. Exploité à deux niveaux, ce programme est d'abord utilisé dans le processus d'accueilintégration dans le cadre de la rétention des employés. Au second niveau, il bonifie et assure la poursuite des apprentissages des employés. Afin de formaliser le processus, un contrat de parrainage dans lequel les deux parties s'engagent dans un processus structuré, ayant des objectifs définis à atteindre, est nécessaire. L'identification des besoins permet de définir le temps qui sera alloué au parrain et au parrainé. Le rôle du parrain est d'écouter, d'enseigner par l'exemple, d'aider l'intervenant parrainé à construire une confiance en ses interventions, de recadrer les problématiques vécues et d'encourager les comportements gagnants. Le parrainage permet aux parrains d'être authentiques face à leur expérience et d'y trouver un sens, tout en offrant aussi aux parrainés des opportunités d'apprentissage et de réalisation. Le parrain partage son sens critique et offre ses encouragements. Le programme de parrainage offre finalement la possibilité aux parrainés de valider leur choix d'intervention et de décisions tout en explorant une manière différente de penser et d'apprendre. Ce programme fait la démonstration d'une culture organisationnelle apprenante axée sur la transmission et le partage des connaissances.

Le parrainage permet aux parrains d'être authentiques face à leur expérience et d'y trouver un sens, tout en offrant aussi aux parrainés des opportunités d'apprentissage et de réalisation. Le parrain partage son sens critique et offre ses encouragements.

En conséquence, le parrainage permet aux intervenants en dépendances de mieux faire face à la complexité de la clientèle, mais aussi d'être mieux préparés à affronter des situations cliniques et d'intervention multiples. En résumé, les parrains sont des alliés importants qui aident les nouveaux à comprendre rapidement «qui fait quoi, où, comment et pourquoi? ». Ils transmettent rapidement la culture de l'organisation et établissent une relation orientée sur l'atteinte d'un jugement professionnel et éthique plus sûr. Il faut donc utiliser le parrainage comme un outil de développement et d'uniformisation des services et de la qualité des interventions dispensées à la clientèle (adulte ou jeune) en dépendance. Ainsi, l'accueil-intégration, le parrainage et la formation furent un cheminement d'apprentissage plutôt qu'une série d'étapes à franchir. 


\section{DISCUSSION}

La mise sur pied d'un programme

d'accueil-intégration et de parrainage s'est traduite par l'efficacité, la motivation, l'engagement des employés au sein de l'organisation et par l'amélioration de la qualité de vie au travail.

Les constats découlant de l'application de ces approches collaboratives se sont révélés positifs. La mise sur pied d'un programme d'accueilintégration et de parrainage s'est traduite par l'efficacité, la motivation, l'engagement des employés au sein de l'organisation et par l'amélioration de la qualité de vie au travail.

Facile à mettre en œuvre, la méthode d'apprentissage fut un moyen économique de former et de permettre une mise à niveau des compétences des intervenants. En plus, de répondre à la volonté d'intégration des nouveaux employés au sein du CRD, elle participe simultanément à la construction des équipes de travail et à l'établissement d'un climat favorable. Certains étaient formateurs lors de ces journées de formation, mais chacun contribuait à l'esprit du travail d'équipe et d'entraide.

Les pairs formateurs ont, quant à eux, permis à leurs collègues de reconnaître leur expertise et l'apport enrichissant de la clientèle jeunesse dans l'organisation. La formation a favorisé un climat d'entraide entre les participants en plus d'humaniser par des exemples concrets et réels l'information transmise. Ce fut l'occasion pour plusieurs intervenants d'obtenir la reconnaissance de leur expérience professionnelle par leurs pairs.

La formation a favorisé un climat d'entraide entre les participants en plus

d'humaniser par des exemples concrets et réels l'information transmise. Ce fut

l'occasion pour plusieurs intervenants d'obtenir la reconnaissance de leur expérience professionnelle par leurs pairs.
Ce type de formation répond aux attentes des intervenants en matière de formations «terrains » et à leur désir d'échange professionnel sur la façon d'intervenir des collègues et de validation de leurs propres savoir-faire. Les journées de «formations par les pairs» ont permis à tous les participants de vivre ensemble une expérience positive. Les commentaires des participants sont très élogieux. De plus, la synthèse des résultats d'évaluation obtenus grâce à des questionnaires d'appréciation, fait état de la grande satisfaction des participants lors des deux journées de formation. Ils ont eu, tout au long de ces formations, un plaisir à inventorier des possibilités d'agir ensemble.

La formation par les pairs a permis de minimiser la résistance aux changements et favorise le développement du sentiment d'appartenance. En effet cette stratégie d'apprentissage a satisfait les besoins exprimés par les intervenants consultés en respectant les principes de la gestion collaborative.

Les formations ont amené les participants à être à l'écoute des idées de l'autre, à développer de nouvelles compétences et à travailler en interaction en partageant des outils et des opinions cliniques dans une équipe interdisciplinaire. En bref, la formation par les pairs a permis, dans le cadre de cette transformation organisationnelle, de construire un socle commun de meilleures pratiques d'intervention auprès de la clientèle.

La formation par les pairs a permis de minimiser la résistance aux changements et favorise le développement du sentiment d'appartenance. En effet cette stratégie d'apprentissage a satisfait les besoins exprimés par les intervenants consultés en respectant les principes de la gestion collaborative. Malgré cette réussite, le défi demeure dans la pérennité de ces approches au sein de l'organisation dans un contexte de mobilité des ressources. 


\section{CONCLUSION}

Grâce à la mise en place et à la consolidation des meilleures pratiques en gestion des ressources humaines, le CRD joue un rôle de précurseur en matière d'accueil, d'intégration et de parrainage au CSSS de Jonquière.

Cette intervention riche en réalisations a permis de développer plusieurs outils de gestion mis en place par l'organisation. L'évaluation actuelle de ces outils démontre l'efficacité et la pertinence de la structuration de l'accueil, de l'intégration des nouveaux employés, du programme de parrainage, de même que du développement des compétences des employés. Ces outils ont inspiré l'élaboration de mesures d'encadrement similaires à la grandeur du CSSS de Jonquière pour les professionnels et techniciens. Conformément à l'atteinte des objectifs de l'arrimage de la clientèle et au délai prévu pour l'exécution des différentes étapes de réalisation, l'évaluation de cette intégration est positive. Grâce à la mise en place et à la consolidation des meilleures pratiques en gestion des ressources humaines, le CRD joue un rôle de précurseur en matière d'accueil, d'intégration et de parrainage au CSSS de Jonquière. À la lumière de l'expérience de la formation par les pairs, retenons la créativité, l'innovation et l'implication collaborative des membres de l'équipe qui se sont avérés comme des éléments de développement. En résumé, une organisation grandit lorsqu'elle prend conscience de la valeur humaine de chaque personne qui la compose.

\section{BIBLIOGRAPHIE}

${ }^{1}$ Bandura, A. (1986). Social Foundations of Thought and Action: A Social Cognitive Theory. N.J: Prentice Hall. Fishbein, M., \& Ajzen, I. (1975). Belief, Attitude, Intention, and Behavior: An Introduction to Theory and Research. Reading, MA: AddisonWesley. ROGERS, C. (1983) L'éducation : une activité qui concerne la personne. Emergences, $\mathrm{n}^{\circ} 3$ : Les Cahiers, I-IV. FREIRE, P. (1970). Pedagogy of the oppressed. New York: Herder and Herder.
${ }^{2}$ Meignant, A. 2004. Manager la formation. Paris : Édition Liaisons.

${ }^{3}$ Bourhis, A. Chênevert, D. 2009. À vos marques, prêts, Gérez! La GRH pour gestionnaires. ERPI.

${ }^{4}$ Meddeb, B. 2003 L'organisation du $\mathrm{III}^{\mathrm{e}}$ millénaire : quelles compétences pour le manager. Revue organisation et Territoires, vol.12 no 1, pp. 5-10.

Notes

á́tudiante diplômée à la maîtrise en gestion des organisations, Université du Québec à Chicoutimi. 


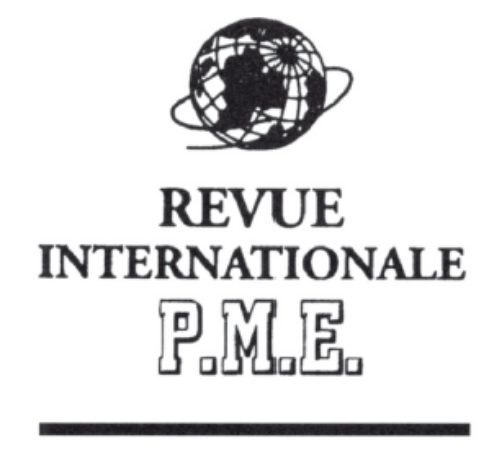

Volume 23, numéro 3-4, 2010

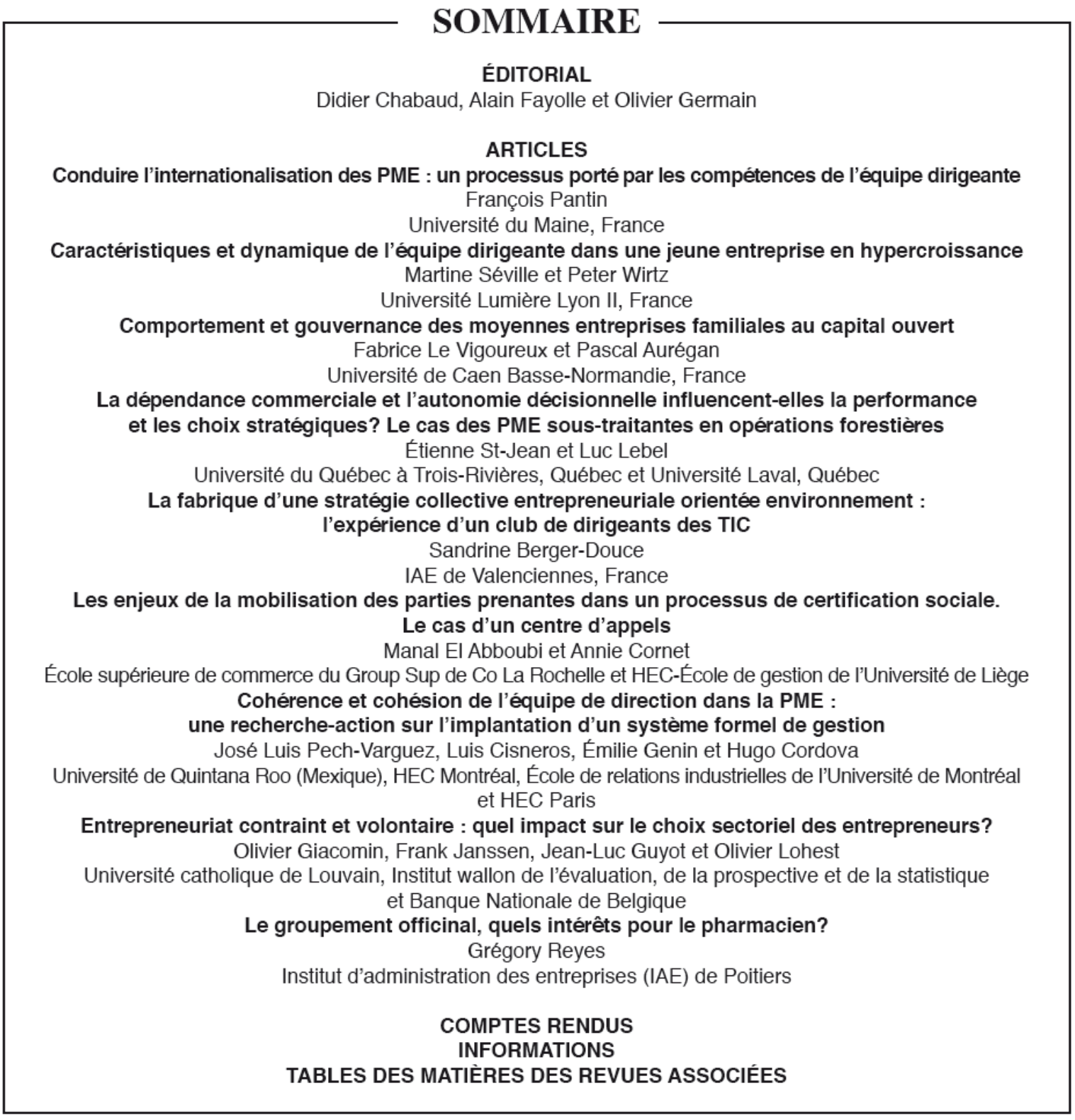

